\title{
Frequency Response Function Analysis of Structures with Fuzzy Modal Damping Parameters
}

\author{
David Moens * , Hilde De Gersem ${ }^{\dagger}$ and Dirk Vandepitte ${ }^{\ddagger}$ \\ K.U.Leuven, Leuven, Belgium
}

\begin{abstract}
The ability to include non-deterministic properties in a numerical simulation process is of great value for a design engineer. In this context, the fuzzy concept has been introduced as a tool for modeling subjective uncertainty in a numerical environment. Recently, a fuzzy finite element methodology has been developed to calculate fuzzy frequency response functions (FRF) of uncertain structures. The methodology for the interval problem at the core of this procedure is based on a new hybrid approach, which consists of a preliminary optimization step, followed by an interval arithmetic procedure. The final envelope FRFs have been proven to give a very good approximation of the actual response range of the interval problem. Initially, the fuzzy method for FRF analysis was developed for damped structures based on the proportional damping principle. This paper introduces a more general approach for the damping uncertainty based on general modal damping. In the proposed procedure, the analyst can define independent modal damping parameter intervals for each mode that is taken into account in the response function. The modal damping intervals are introduced directly into the procedure. This means that there is no extra preliminary optimization necessary. It is shown how the introduction of the modal damping intervals influences the interval arithmetical procedure that calculates the envelope response function based on the modal superposition principle. In order to validate the procedure, it is applied to a realistic model with fuzzy modal damping parameters.
\end{abstract}

\section{Introduction}

Non-deterministic approaches are gaining popularity in the field of numerical analysis. This is mainly caused by the growing computational capabilities of modern computers, which has lead to an increase in the degree of complexity of models used for numerical analysis. In the simulation of these detailed models, not all numerical properties are always readily available. Especially during the design of a product, numerical descriptions of the model details often are not available or only partially known. Therefore, the ability to include non-deterministic properties is of great value for a design engineer. On the one hand, nondeterministic analysis enables a reliability assessment, which includes the uncertain aspects of the design in the numerical computation. On the other hand, the design can be optimized for robust behavior under varying external influences.

The probabilistic concept is by far the most popular non-deterministic modeling tool. Over the past decade, however, some criticism arises on the general application of the probabilistic concept. It has been shown that under specific conditions, non-probabilistic approaches could be equally valuable but more efficient than the classic probabilistic approach. ${ }^{1}$ Especially for analyses with limited available objective information on the uncertain model properties, the limited usefulness of a subjective probabilistic analysis result often does not justify its computational cost. In this context, the fuzzy concept has been introduced in the world of non-deterministic numerical simulations. Its main advantage is its capability to model limited or subjective knowledge. Furthermore, the fuzzy concept has led to some new possibilistic approaches for product reliability analysis and design optimization. ${ }^{2}$

${ }^{*}$ IWT Post-doctoral Research Fellow, Dept. of Mechanical Engineering, Celestijnenlaan 300B, 3001 Heverlee, Belgium, AIAA member

${ }^{\dagger}$ FWO Research Fellow, Dept. of Mechanical Engineering, Celestijnenlaan 300B, 3001 Heverlee, Belgium

${ }^{\ddagger}$ Full Professor, Dept. of Mechanical Engineering, Celestijnenlaan 300B, 3001 Heverlee, Belgium, AIAA member 
The application of the fuzzy concept in the framework of finite element (FE) analysis has been studied in different areas. ${ }^{3-5}$ The goal of a fuzzy FE analysis is to obtain a fuzzy description of a certain outcome of a FE analysis, starting from fuzzy descriptions of all non-deterministic input properties. Numerically, the fuzzy $\mathrm{FE}$ analysis comes down to an iterative procedure, consisting of a sequence of interval FE analyses. This is generally referred to as the $\alpha$-sublevel technique. Recently, a fuzzy FE methodology has been developed to calculate fuzzy frequency response functions (FRF) of uncertain structures. ${ }^{6}$

The first development in the area of the fuzzy FRF analysis was on undamped structures. The method later was extended to damped structures based on the proportional damping principle. ${ }^{7}$ This extension is numerically beneficial since through the proportional damping, there is no extra modal parameter involved in the analysis. The modal damping is directly calculated as a linear combination of the modal mass and stiffness values. For the fuzzy FRF procedure, this means that the interval optimization results of the preliminary step of the undamped case can be directly transformed into modal damping intervals by applying this linear combination on the modal mass and stiffness intervals. However, whereas the concept of proportional damping is very interesting from a numerical point of view, it poses an important restriction on the damping model. Even though the modal damping is contained within an interval, the actual modal damping taken into account in a specific model will always remain implicitly coupled to the modal stiffness and mass in that particular case. This means that over all models, the modal damping will indeed vary in its interval, but it will do so proportionally coupled to modal stiffness and mass. In dynamic analysis, damping is a model property that is commonly subject to a high amount of uncertainty. Since the proportional damping concept fails to analyze the influence of this damping uncertainty independently from mass or stiffness variations, an extension of the analysis procedure towards independent modal damping would substantially improve the value of fuzzy FRF analysis in structural dynamics. This paper therefore introduces a new methodology for fuzzy FRF calculations based on general modal damping.

This paper first describes the basic properties of the fuzzy finite element analysis in section [I] This section also summarizes some general numerical aspects of the fuzzy FE methodology. Section III then gives a general overview of the basic undamped procedure as developed by the authors. ${ }^{7}$ The theoretical extension to general modal damping is given in section IV] The presented algorithm is finally validated on a numerical example in section $\mathrm{V}$.

\section{The Fuzzy Finite Element Method}

\section{A. Fuzzy Sets}

The concept of fuzzy sets, introduced by Zadeh ${ }^{8}$ in 1965, has gained an increasing popularity during the last two decades. Its most important property is that it is capable of describing linguistic and therefore incomplete information in a non-probabilistic manner. Whereas a classical set clearly distinguishes between members and non-members, a fuzzy set introduces a degree of membership, represented by the membership function. For a fuzzy set $\tilde{x}$, the membership function $\mu_{\tilde{x}}(x)$ describes the grade of membership to the fuzzy set for each element $x$ in the domain $X$ :

$$
\tilde{x}=\left\{\left(x, \mu_{\tilde{x}}(x)\right) \mid(x \in X)\left(\mu_{\tilde{x}}(x) \in[0,1]\right)\right\}
$$

If $\mu_{\tilde{x}}(x)=1, x$ is definitely a member of the set $\tilde{x}$, whereas if $\mu_{\tilde{x}}(x)=0, x$ is definitely not a member of the set $\tilde{x}$. For all $x$ with $0<\mu_{\tilde{x}}(x)<1$, the membership is not certain. The most frequently applied membership function shapes are the triangular and Gaussian shape, as illustrated in figure 1 .

In the fuzzy finite element method as introduced by Rao et al., ${ }^{3}$ the uncertain model properties are modeled by fuzzy sets through the concept of membership functions. These define the degree of possibility that a specific numerical value belongs to the set of values corresponding to an uncertain model property. The goal of a fuzzy FE analysis is to obtain a fuzzy description of a specific numerical result of the FE analysis, starting from fuzzy descriptions of all non-deterministic input properties.

\section{B. Fuzzy Numerical Analysis}

The most common procedure for the calculation of output membership function starting from input fuzzy sets is based on the $\alpha$-level strategy. This approach subdivides the membership function range into a number of $\alpha$-levels. The intersection with the membership function of the input uncertainties at each $\alpha$-level results in an interval, denoted by a boldface symbol $\mathbf{x}_{i, \alpha}=\left[\underline{x}_{i}, \bar{x}_{i}\right]_{\alpha}$. With these input intervals, an interval analysis 

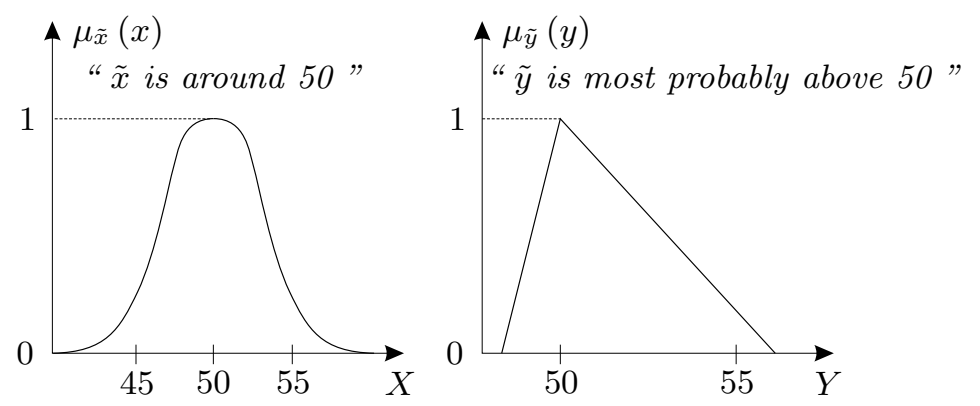

Figure 1. Common membership functions used to represent uncertain information

is performed, resulting in an interval for the analysis result at the considered $\alpha$-level. Finally, the fuzzy solution is assembled from the resulting intervals at each sublevel. Figure 2 clarifies this procedure for a function of two triangular fuzzy parameters.

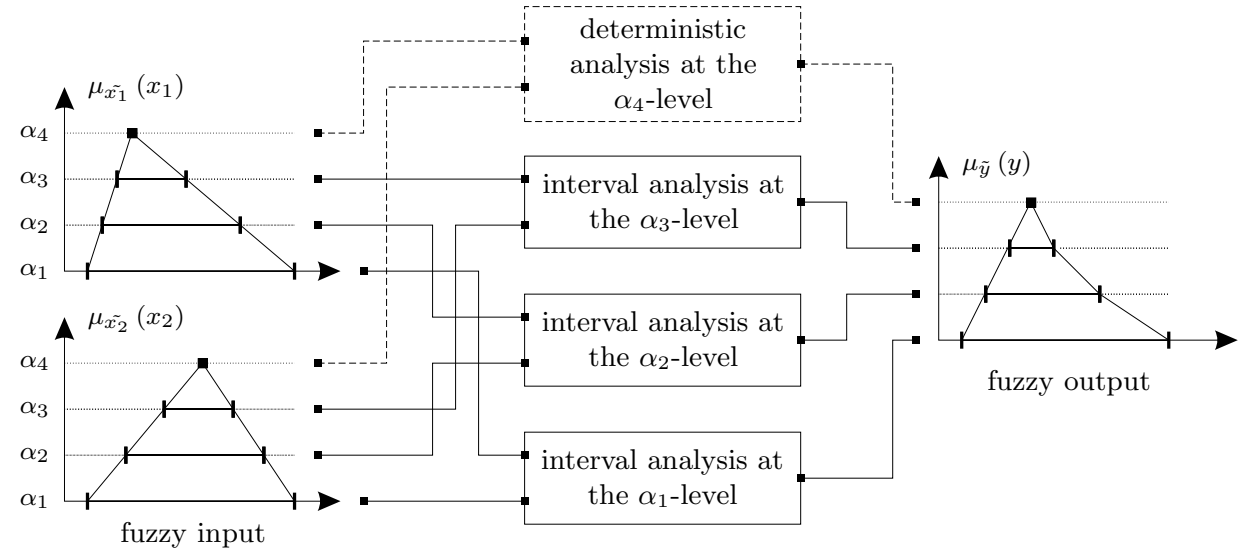

Figure 2. $\alpha$-level strategy with $4 \alpha$-levels, for a function of two triangular fuzzy parameters

The fuzzy finite element technique consists of the application of the $\alpha$-level strategy on the numerical procedure of the deterministic FE analysis. Through this procedure, the fuzzy FE analysis can be interpreted as a large-scale sensitivity analysis, which can be used to study the combined effect of the interval bounds of design variables on critical design properties. For instance, by placing membership functions on the design properties subject to tolerances, the effect of their range on the design behavior can be analyzed. If in the fuzzy outcome of the analysis, an allowable range is identified at a certain membership level, the corresponding input intervals at this level represent acceptable tolerances on the analyzed design properties. This is one of the possible applications of fuzzy analysis in a design engineering context (see Moens et al. ${ }^{9}$ for a more comprehensive overview of fuzzy analysis for non-deterministic analysis in design).

From a numerical point of view, the solution at each $\alpha$-level of the interval problem corresponding to the considered FE analysis forms the core of this approach. A number of general interval solution strategies have been proposed as the basis for fuzzy FE implementations. The most popular strategy is the interval arithmetic approach. In this approach, all basic operations in the deterministic algorithm are substituted with their interval arithmetic counterparts:

$$
\begin{aligned}
\mathbf{a}+\mathbf{b} & =[\underline{a}+\underline{b}, \bar{a}+\bar{b}] \\
\mathbf{a}-\mathbf{b} & =[\underline{a}-\bar{b}, \bar{a}-\underline{b}] \\
\mathbf{a} \times \mathbf{b} & =[\min (\underline{a} \times \underline{b}, \underline{a} \times \bar{b}, \bar{a} \times \underline{b}, \bar{a} \times \bar{b}) \max (\underline{a} \times \underline{b}, \underline{a} \times \bar{b}, \bar{a} \times \underline{b}, \bar{a} \times \bar{b})] \\
\mathbf{a} / \mathbf{b} & =\mathbf{a} \times\left[\frac{1}{\bar{b}}, \frac{1}{\underline{b}}\right], \text { if } 0 \notin \mathbf{b}
\end{aligned}
$$

Whereas this procedure is computationally very inexpensive, this approach tends to substantially overes- 
timate the interval outcome of each operation that is substituted with the corresponding interval operation. This generally causes a very high amount of conservatism in the final analysis result. Therefore, other techniques like the transformation method ${ }^{10}$ and a global optimization strategy ${ }^{3}$ have been proposed in this context. However, up to now, none of these approaches has really become a standard because all of these exhibit specific disadvantages when applied to realistically sized models. This paper applies a newly developed approach which combines the global optimization with the interval arithmetic approach, resulting in a hybrid strategy with an acceptable computational cost and limited amount of conservatism in its result.

\section{The Fuzzy Finite Element Method for FRF analysis}

The fuzzy finite element method for the calculation of frequency response functions (FRFs), developed by the authors, combines the concept of fuzzy sets with the modal superposition principle. The methodology is based on a new hybrid approach, which consists of a preliminary optimization step, followed by an interval arithmetic procedure. In the first part of this procedure, the optimization is used to translate the interval properties defined on the FE model to the exact interval modal stiffness and mass parameters of the structure. The calculation of the envelope FRFs in the second part is done by applying the interval arithmetic equivalent of the modal superposition procedure on these interval modal parameters. This procedure neutralizes all conservatism in the matrix assembly phase, since it directly uses the modal parameters as goal functions in the optimization part. The final envelope FRFs have been proven to contain only a very limited amount of conservatism. A brief overview of the basic principles of the method is given in this section. The complete mathematical description of the method can be found in Moens et al. ${ }^{6,7}$

\section{A. The deterministic modal superposition principle}

For undamped structures, the deterministic modal superposition principle states that, considering the first $n_{\text {modes }}$ modes, the frequency response function between degrees of freedom $j$ and $k$ equals:

$$
F R F_{j k}=\sum_{i=1}^{n_{\text {modes }}} F R F_{j k}^{i}=\sum_{i=1}^{n_{\text {modes }}} \frac{\phi_{i_{k}} \phi_{i_{j}}}{\left\{\phi_{i}\right\}^{T}[K]\left\{\phi_{i}\right\}-\omega^{2}\left\{\phi_{i}\right\}^{T}[M]\left\{\phi_{i}\right\}}
$$

with $\left\{\phi_{i}\right\}$ the $i^{\text {th }}$ eigenvector of the system and $\phi_{i_{j}}$ the $j^{\text {th }}$ component of the $i^{\text {th }}$ eigenvector. Simplification of Eq. (6) yields:

$$
F R F_{j k}=\sum_{i=1}^{n_{\text {modes }}} \frac{1}{\hat{k}_{i}-\omega^{2} \hat{m}_{i}}
$$

with $\hat{k}_{i}$ and $\hat{m}_{i}$ the modal parameters defined as:

$$
\begin{aligned}
\hat{k}_{i} & =\frac{\left\{\phi_{i}\right\}^{T}[K]\left\{\phi_{i}\right\}}{\phi_{i_{j}} \phi_{i_{k}}} \\
\hat{m}_{i} & =\frac{\left\{\phi_{i}\right\}^{T}[M]\left\{\phi_{i}\right\}}{\phi_{i_{j}} \phi_{i_{k}}}
\end{aligned}
$$

\section{B. Undamped Fuzzy Finite Element FRF analysis}

The modal superposition principle for undamped structures has been translated into an interval finite element method for FRF analysis. Figure 3 gives a graphical overview of the translation of the deterministic algorithm into an interval procedure. On the left-hand side is the deterministic algorithm as described in the previous section. On the right-hand side is the same procedure translated to an equivalent interval algorithm.

The interval method consists of the calculation of the result ranges of the subfunctions appearing in the consecutive steps of the deterministic algorithm. Therefore, the deterministic algorithm is split into three subfunctions. In the first step, step 1.1, the modal stiffness $\hat{k}_{i}$ and mass $\hat{m}_{i}$ are calculated for each considered mode. Step 1.2 then consists of the calculation of the modal FRF contributions $F R F_{j k}^{i}$. Step 1.1 and 1.2 have to be performed for each mode that is taken into consideration in the modal superposition. Therefore, it is referred to as the modal part. In step 2, the superposition is performed by a summation of the modal FRF contributions. 


\begin{tabular}{|c|c|}
\hline deterministic algorithm & interval algorithm \\
\hline step 1.1 & step 1.1 \\
\hline$\hat{k}_{i}=\frac{\left\{\phi_{i}\right\}^{T}[K]\left\{\phi_{i}\right\}}{\phi_{i_{j}} \phi_{i_{k}}}=\frac{1}{\phi_{i_{j}}^{K} \phi_{i_{k}}^{K}}$ & $\left\langle\hat{k}_{i}\right\rangle=\left[\min _{\{x\} \in\{\mathbf{x}\}}\left(\phi_{i_{j}}^{K} \phi_{i_{k}}^{K}\right), \max _{\{x\} \in\{\mathbf{x}\}}\left(\phi_{i_{j}}^{K} \phi_{i_{k}}^{K}\right)\right]^{-}$ \\
\hline$\hat{m}_{i}=\frac{\left\{\phi_{i}\right\}^{T}[M]\left\{\phi_{i}\right\}}{\phi_{i_{j}} \phi_{i_{k}}}=\frac{1}{\phi_{i_{j}}^{M} \phi_{i_{k}}^{M}}$ & $\left\langle\hat{m}_{i}\right\rangle=\left[\min _{\{x\} \in\{\mathbf{x}\}}\left(\phi_{i_{j}}^{M} \phi_{i_{k}}^{M}\right), \max _{\{x\} \in\{\mathbf{x}\}}\left(\phi_{i_{j}}^{M} \phi_{i_{k}}^{M}\right)\right]^{-}$ \\
\hline step 1.2 & step 1.2 \\
\hline$F R F_{j k}^{i}=\frac{1}{\hat{k}}$ & $\left\langle F R F_{j k}^{i}\right\rangle=\frac{1}{1 \hat{i}}$ \\
\hline $\begin{array}{l}\text { step } 2 \\
\qquad F R F_{j k}=\sum_{i=1}^{n} F R F_{j k}^{i}\end{array}$ & $\left\langle F R F_{j k}\right\rangle=\sum_{i=1}^{n}\left\langle F R F_{j k}^{i}\right\rangle$ \\
\hline
\end{tabular}

Figure 3. Translation of the deterministic modal superposition algorithm to an equivalent IFE procedure

The interval procedure follows the same outline as the deterministic algorithm. Each step now concentrates on the derivation of the range of the subfunctions in the deterministic algorithm:

step 1.1 For all $n_{\text {modes }}$ taken into account, the ranges of possible values that the modal stiffness and mass can adopt have to be determined, taking into account that the uncertain parameters in $\{x\}$ can vary within their respective intervals. These correct ranges of the modal parameters denoted by $\left\langle\hat{k}_{i}\right\rangle$ and $\left\langle\hat{m}_{i}\right\rangle$ are determined using a minimization and maximization over the uncertain interval space $\{\mathbf{x}\}$. For numerical convenience, the global optimization is performed on the inverted modal parameters, after which the obtained intervals are inverted in order to obtain the actual modal parameter ranges:

$$
\begin{aligned}
\left\langle\hat{k}_{i}\right\rangle & =\left[\min _{\{x\} \in\{\mathbf{x}\}}\left(\phi_{i_{j}}^{K} \phi_{i_{k}}^{K}\right), \max _{\{x\} \in\{\mathbf{x}\}}\left(\phi_{i_{j}}^{K} \phi_{i_{k}}^{K}\right)\right]^{-1} \\
\left\langle\hat{m}_{i}\right\rangle & =\left[\min _{\{x\} \in\{\mathbf{x}\}}\left(\phi_{i_{j}}^{M} \phi_{i_{k}}^{M}\right), \max _{\{x\} \in\{\mathbf{x}\}}\left(\phi_{i_{j}}^{M} \phi_{i_{k}}^{M}\right)\right]^{-1}
\end{aligned}
$$

with $\left\{\phi_{i}^{K}\right\}$ and $\left\{\phi_{i}^{M}\right\}$ the stiffness and mass normalized eigenvectors of the system.

step 1.2 The modal envelope FRF is calculated by substituting the ranges of the modal parameters in the deterministic expression of the modal FRF contribution:

$$
\left\langle F R F_{j k}^{i}\right\rangle=\frac{1}{\left\langle\hat{k}_{i}\right\rangle-\omega^{2}\left\langle\hat{m}_{i}\right\rangle}
$$

This is an analytical procedure performed using the interval arithmetic approach.

step 2 Finally, the total interval FRF is obtained by the summation of the contributions of all considered modes:

$$
\left\langle F R F_{j k}\right\rangle=\sum_{i=1}^{n}\left\langle F R F_{j k}^{i}\right\rangle
$$

Also this final step is performed using interval arithmetics. 


\section{Eigenvalue Interval Correction}

The method as described above can be enhanced based on a graphical interpretation of the modal part of the interval algorithm. For each mode, we consider the domain of modal mass and stiffness pairs that can be achieved by considering the complete range of models defined by the interval uncertainty space $\{\mathbf{x}\}$ :

$$
\left\langle\hat{k}_{i}, \hat{m}_{i}\right\rangle=\left\{\left(\hat{k}_{i}, \hat{m}_{i}\right) \mid(\{x\} \in\{\mathbf{x}\})\right\}
$$

This domain defines a bounded area in a $\hat{k}_{i}, \hat{m}_{i}$-workspace. The exact bounds of this domain however, are generally unknown. The modal part of the interval algorithm now can be interpreted in this workspace. From the optimization as described for step 1.1, it is clear that the calculated ranges on the modal parameters $\left\langle\hat{k}_{i}\right\rangle$ and $\left\langle\hat{m}_{i}\right\rangle$ represent a rectangular approximation of the actual $\left\langle\hat{k}_{i}, \hat{m}_{i}\right\rangle$-domain. Therefore, this method is referred to as the Modal Rectangle (MR) method. Figure 4 shows a general $\left\langle\hat{k}_{i}, \hat{m}_{i}\right\rangle$-domain and its approximation using the MR method.

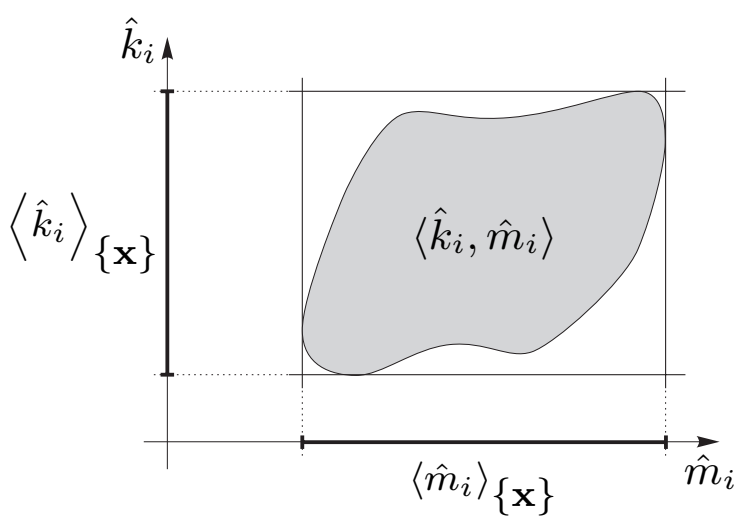

Figure 4. Graphical illustration of a mode's $\left\langle\hat{k}_{i}, \hat{m}_{i}\right\rangle$-domain and its approximation using the modal rectangle method.

The interval arithmetic procedure for the calculation of the modal envelope FRF contributions in step 1.2 can be interpreted in the same graphical domain. The goal in this step is to derive the bounds on the deterministic modal FRF, taking into account that $\hat{k}_{i}$ and $\hat{m}_{i}$ can be anywhere inside their intervals. By considering the modal FRF contribution as defined in Eq. $\sqrt{12}$ as an analytical function of $\hat{k}_{i}$ and $\hat{m}_{i}$, the bounds on this function over the modal rectangle have to be determined. It has been shown that this can be done analytically for the complete frequency domain, by considering only the function evaluations at the upper left and the lower right corner points of the rectangle.

Based on these observations, it becomes clear that the calculation based on the modal rectangle introduces conservatism in the procedure if the actual $\left\langle\hat{k}_{i}, \hat{m}_{i}\right\rangle$-domain differs strongly from the approximate rectangle. This is often the case, as these parameters are actually coupled through the global system and generally show a high degree of correlation. The proposed enhancement therefore focusses on an improved approximation of the $\left\langle\hat{k}_{i}, \hat{m}_{i}\right\rangle$-domain. This can be achieved by using information on the eigenvalue ranges, which can be obtained using an additional eigenvalue optimization step in the modal part of the algorithm. It is clear that an eigenvalue interval $\boldsymbol{\lambda}_{\boldsymbol{i}}$ introduces an extra restriction on the quotient of possible combinations of the modal parameters. This restriction is mathematically expressed as:

$$
\underline{\lambda_{i}} \leq \frac{\hat{k}_{i}}{\hat{m}_{i}} \leq \overline{\lambda_{i}}
$$

Graphically, the eigenvalue bounds represent the lines through the origin of the $\hat{k}_{i}, \hat{m}_{i}$-space which are tangent to the actual $\left\langle\hat{k}_{i}, \hat{m}_{i}\right\rangle$-domain. These lines are extra delimiters for the $\left\langle\hat{k}_{i}, \hat{m}_{i}\right\rangle$-domain approximation, and therefore give rise to an improved $\left\langle\hat{k}_{i}, \hat{m}_{i}\right\rangle$-domain approximation as illustrated in figure 5 . This domain is referred to as the Modal Rectangle with Eigenvalue interval correction (MRE).

It has been shown that the conservatism in the modal envelope FRF contributions derived in step 1.2 can now be substantially reduced by considering the MRE domain instead of the MR domain as area of

$$
6 \text { of } 11
$$




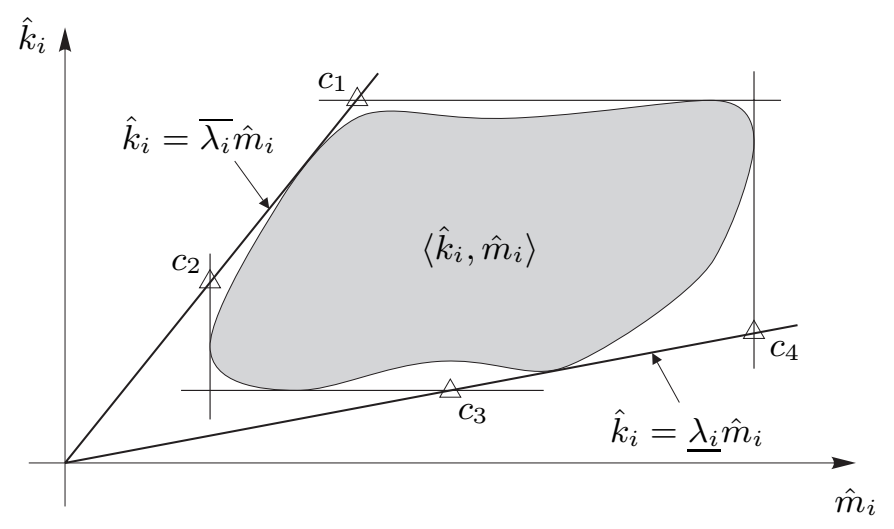

Figure 5. Effect of the introduction of the exact eigenvalue interval in the $\left\langle\hat{k}_{i}, \hat{m}_{i}\right\rangle$-domain approximation of a positive mode.

possible modal parameter pairs. The modal envelope FRF contributions can be determined analytically by calculating the deterministic modal FRFs at the vertex points of the MRE-domain (indicated with $c_{i}$, $i=1 \ldots 4$ in figure 5. It has been shown that only considering these vertex points leads to a close and guaranteed outer approximation of the actual modal envelope FRF contribution. ${ }^{7}$

\section{Damped Fuzzy Finite Element FRF analysis}

Uncertain modal damping is now introduced in the above procedure. The fuzzy uncertainty is defined directly on the modal damping parameters. This means that the existing interval algorithm for the calculation of undamped envelope FRFs has to be extended in order to enable the introduction of a modal damping interval $\hat{\mathbf{c}}_{\mathbf{i}}$ in the procedure. The modal damping interval appears directly in step 1.1 of the modal part of the algorithm. Since the uncertainty has been defined on the modal parameter itself, no extra optimization is necessary in this step. The extension of the method therefore is concentrated in step 1.2 and step 2 of the procedure, which are treated now respectively in sections $\mathrm{A}$ and $\mathrm{B}$.

\section{A. Damped Modal Envelope FRF Contributions (step 1.2)}

In the damped case, the real and imaginary part of the modal FRF contributions are considered separately. Applying the deterministic modal superposition principle and splitting the modal FRF into its real and imaginary parts yields:

$$
\begin{aligned}
\Re\left(F R F_{j k}^{i}\right) & =\frac{\hat{k}_{i}-\omega^{2} \hat{m}_{i}}{\left(\hat{k}_{i}-\omega^{2} \hat{m}_{i}\right)^{2}+\hat{c}_{i}^{2} \omega^{2}} \\
\Im\left(F R F_{j k}^{i}\right) & =\frac{-\hat{c}_{i} \omega}{\left(\hat{k}_{i}-\omega^{2} \hat{m}_{i}\right)^{2}+\hat{c}_{i}^{2} \omega^{2}}
\end{aligned}
$$

In step 1.2 of the interval algorithm, the ranges of these parts have to be derived, given that the modal parameters are within the bounds derived in step 1.1. The procedure for this can be based on the graphical interpretation as described for the undamped case in section $\mathrm{C}$. The changes with respect to the undamped case are the following:

- for each mode, the modal envelope FRF on both the real and imaginary part have to be calculated:

$$
\begin{aligned}
& \left\langle\Re\left(F R F_{j k}^{i}\right)\right\rangle \\
& \left\langle\Im\left(F R F_{j k}^{i}\right)\right\rangle
\end{aligned}
$$


- Both parts of the complex modal FRF can be regarded as a function of the three modal parameters $\hat{k}_{i}, \hat{m}_{i}$ and $\hat{c}_{i}$. Therefore, the modal domain that has to be considered in this step now becomes three-dimensional.

First, a domain of possible modal parameter triplets has to be defined. For the modal mass and modal stiffness, the MRE-domain is considered. The introduction of the modal damping interval directly on the modal level defines an interval on the $\hat{c}_{i}$ axis. Combination of this interval with the MRE-domain results in the domain as illustrated in figure 6

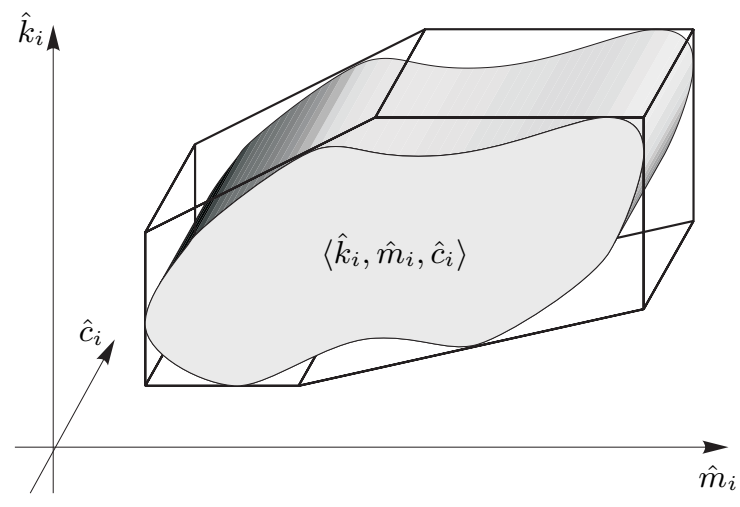

Figure 6. MRE $\left\langle\hat{k}_{i}, \hat{m}_{i}, \hat{c}_{i}\right\rangle$-domain approximation after introduction of a modal damping interval

Based on the three-dimensional domain, the bounds on the real and imaginary part can be derived by considering both parts given in Eqs. (16 17) as explicit functions of the modal parameters. It can be shown that the bounds on the real and imaginary part follow directly from the analytical analysis of these functions only on the horizontal and vertical bounding lines of the domain depicted in figure 6 . This results in a fast analytical procedure for the determination of the real and imaginary parts of the modal envelope FRFs.

\section{B. Damped Total Envelope FRF (step 2)}

The real and imaginary parts of the modal envelope FRF contributions as derived in the previous section now have to be summed in order to obtain the total envelope FRF. Additionally, these total envelope FRFs are converted into amplitude and phase envelopes on the total FRF, denoted respectively as $\left\langle\left\|F R F_{j k}\right\|\right\rangle$ and $\left\langle\varphi\left(F R F_{j k}\right)\right\rangle$. First, the total real and imaginary envelope FRFs are calculated:

$$
\begin{aligned}
\left\langle\Re\left(F R F_{j k}\right)\right\rangle & =\sum_{i=1}^{n}\left\langle\Re\left(F R F_{j k}^{i}\right)\right\rangle \\
\left\langle\Im\left(F R F_{j k}\right)\right\rangle & =\sum_{i=1}^{n}\left\langle\Im\left(F R F_{j k}^{i}\right)\right\rangle
\end{aligned}
$$

This summation is easily implemented because the modal response contributions all describe a finite interval both for the real and the imaginary part of the FRF. The result of the summation is an interval range for the real and imaginary part of the complex response for every frequency. These interval ranges define a rectangle in the complex space in which the response vector is contained. Based on this rectangle, an approximation of the amplitude range of the complex response is obtained by taking the points on the rectangle which are respectively the nearest and most distant from the origin. The phase range is derived in a similar way. Figure 7 illustrates this procedure.

\section{Numerical Example: Solar panel with geometric and damping uncertainties}

\section{A. Problem description}

This test case is a single panel in a satellite solar array, in the stowed condition. A full solar array wing consists of a series of articulated panels that are stowed during launch, and that unfold in orbit. During

$$
8 \text { of } 11
$$




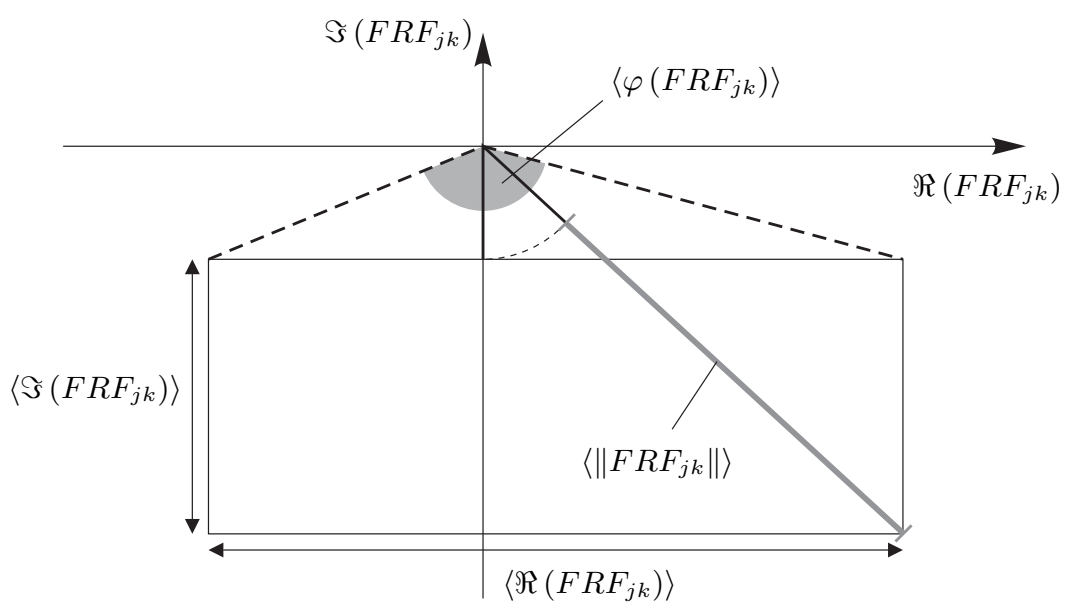

Figure 7. Conversion of the real and imaginary FRF range to the amplitude and phase FRF range

launch, the panels are firmly attached to the satellite structure in a number of holddown points. Load input is a base excitation, with vibrations of the launcher transmitted to the solar array through the satellite. Design criteria are applied on the magnitude of transverse displacements of the panel tips, with tips of adjacent panels in the stack not hitting each other to avoid damage to the solar cells.

In this test case, which is kindly provided by Dutch Space, Leiden (NL), a single panel in a solar array is considered. The panel is a honeycomb structure with six stiffened areas around the holddown points. In these stiffened areas, the density of the honeycomb core is higher to withstand local loads. An important problem encountered during the design phase of the solar panel is the sizing of the diameter of these denser areas, with the potting compound. Two conflicting design criteria have to be considered. On the one hand, the tip displacements must be kept within allowable bounds in order to avoid damage to the solar cells. This design criterion can be achieved by enlarging the stiff potting compound zones. On the other hand, as the material of these stiffened zones is 4.5 times heavier than the surrounding material, the potting compound zones should not be oversized, in order to keep the weight of the satellite as low as possible. In order to find a compromise between the two design criteria, the influence of the diameter of these potting compound zones on the dynamic displacements of the tips of a single panel of the folded structure is investigated using a fuzzy finite element analysis. In this analysis, modal damping uncertainty is included as an additional uncertainty that creates a scatter on the obtained dynamic responses.

The FE model, developed by Dutch Space, consists of a $2.25 \mathrm{~m}$ by $2.732 \mathrm{~m}$ panel built with 916 composite plate elements, and it contains 6018 degrees of freedom. In figure 8 the six potting compound zones can be recognized as the six circular zones. The variation of the diameter of the stiffened zones has been modeled in the FE model by a number of successive concentric rings of plate elements. By changing the material properties of these plate elements, the diameter of the stiff potting compound zones is altered in a discrete way.

\section{B. Damped fuzzy FRF analysis}

A damped fuzzy FRF analysis is performed with the size of the six potting compound zones and the modal damping as uncertain parameters. Figure 9 shows the membership function that is used for all six stiffened zones. The modal damping intervals are such that the damping ratios all vary within the interval $[1 \%, 2 \%]$. This interval is kept constant at all $\alpha$-levels. The tip displacement of the solar panel is taken as response quantity while the dynamic force is introduced as an imposed displacement base excitation perpendicular to the panel (cfr. figure 8). The imposed displacement load is specified using the large mass method. During the analysis, the first 20 modes are taken into account, covering a frequency range up to $240 \mathrm{~Hz}$. The MRE method is applied on the interval levels of the fuzzy analysis.

The resulting fuzzy FRF on the amplitude of the tip displacement of the panel is shown in figure 10 . Only the membership evolution of the upper bound of the FRF is illustrated as the lower bound is very small for a large part of the frequency domain. The figure clearly shows the evolution of the tip displacement with respect to the size of the design uncertainty interval on the dimension of the stiffened zones. In this 


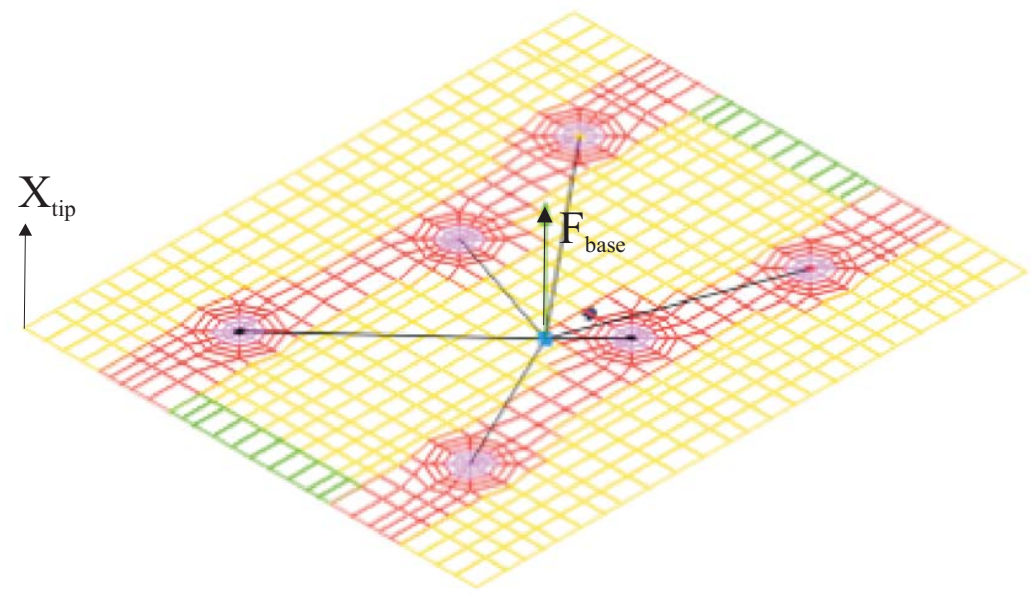

Figure 8. Finite element model of a single panel of the folded structure (model development by Dutch Space)

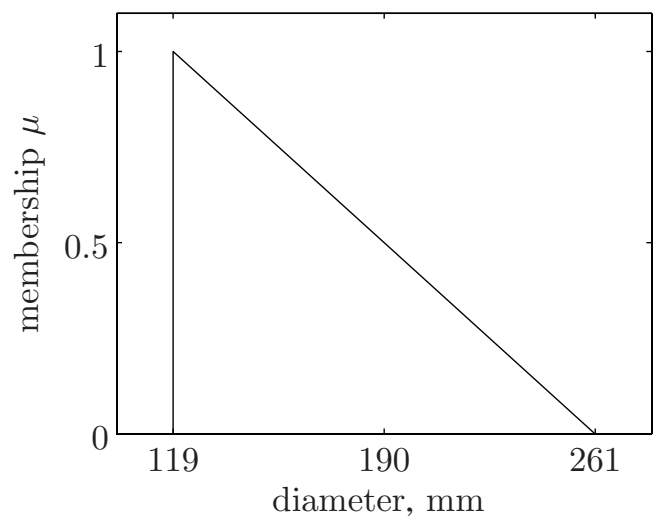

Figure 9. Membership function for the diameter of the potting compound zones of the solar panel

case, the resonances in the region around $65 \mathrm{~Hz}$ clearly determine the maximum response level that can be reached in the observed frequency domain. The fuzzy analysis in this case reveals that exactly in this region, the impact of the uncertainty is relatively high. This means that the designer can base the choice of the diameter of the potting compound zones on the fuzzy FRF by selecting an acceptable response level. The maximal membership value that is reached for this response level over the analyzed frequency domain determines the acceptable $\alpha$-level. Based on the principle of the $\alpha$-level strategy, the designer now can conclude that choosing the diameter of the stiffened zones inside the corresponding interval on the input membership function will lead to a safe design.

\section{Conclusion}

This paper shows how the algorithm for the calculation of fuzzy frequency response functions of undamped structures can be extended to structures with uncertain modal damping properties. The development starts from the existing modal rectangle method with eigenvalue interval correction as developed for the undamped envelope FRF analysis. The additional uncertainty on the damping is introduced as an extra parameter in the modal part of the algorithm. It is shown how the definition of an uncertainty interval on these modal damping parameters affects the algorithm. In order to perform the damped response analysis, the algorithm is split up in two parts, considering respectively the real and imaginary part of the response. The envelopes 


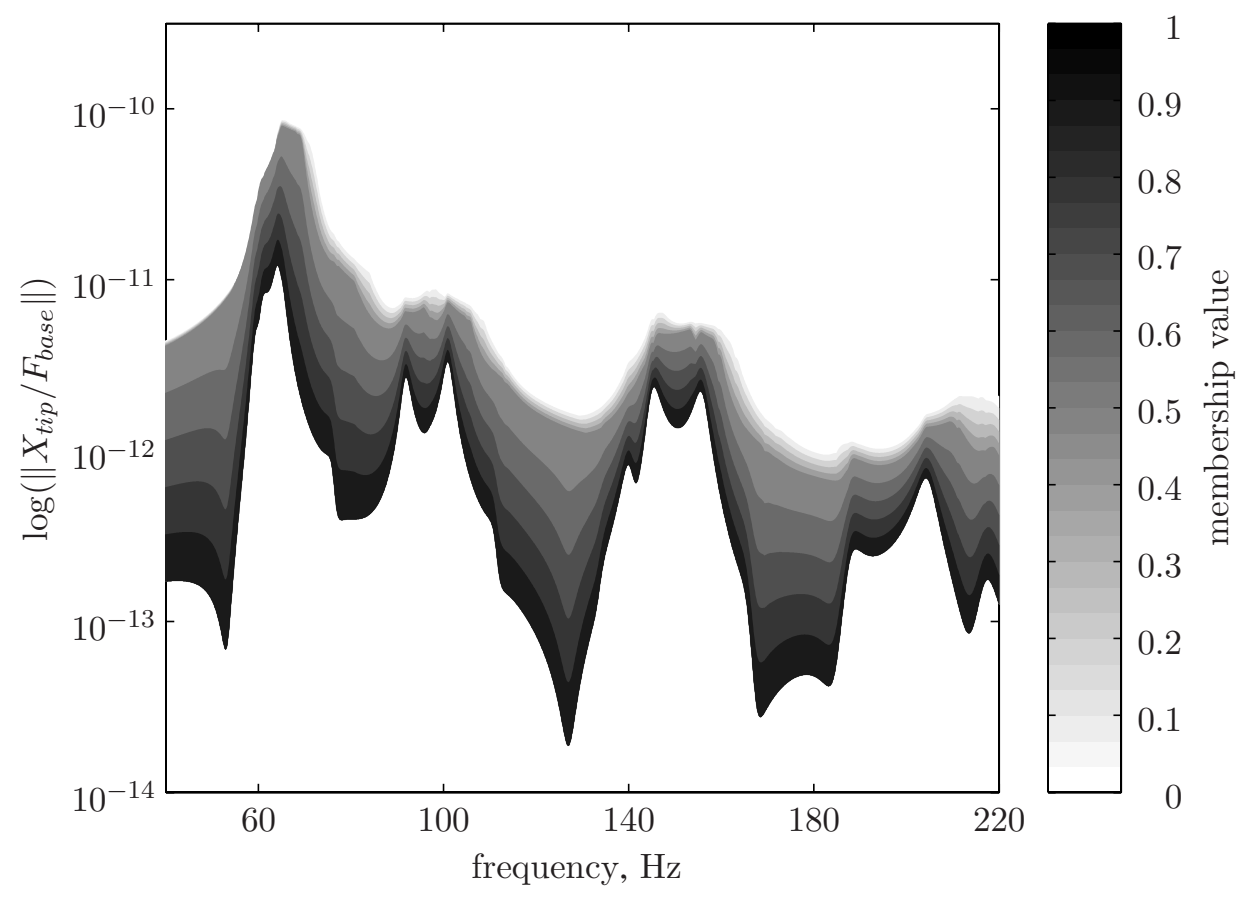

Figure 10. Damped fuzzy FRF (upper bound) of a single solar panel

on both parts of the modal contributions are obtained by adding a third dimension to the considered modal parameter domain. This finally results in a computationally efficient procedure for fuzzy uncertainty analysis of damped response functions. The method is illustrated on a realistic test case.

\section{Acknowledgments}

The test case of the solar panel was kindly made available by J. Wijker of Dutch Space, Leiden (NL). The research of D. Moens is funded by a post-doctoral fellowship from the Institute for the promotion of Innovation by Science and Technology in Flanders (IWT - Vlaanderen), Brussel. H. De Gersem is research assistant of the Fund for Scientific Research - Flanders (Belgium) (F.W.O.-Vlaanderen).

\section{References}

${ }^{1}$ Zingales, M. and Elishakoff, I., "Anti-Optimization Versus Probability in an Applied Mechanics Problem: Vector Uncertainty," Transactions of the ASME, Journal of Applied Mechanics, Vol. 67, 2000, pp. 472-483.

${ }^{2}$ Ben Haim, Y., "A Non-Probabilistic Measure of Reliability of Linear Systems based on Expansion of Convex Models," Structural Safety, Vol. 17, 1995, pp. 91-109.

${ }^{3}$ Rao, S. and Sawyer, P., "Fuzzy Finite Element Approach for the Analysis of Imprecisely Defined Systems," AIAA Journal, Vol. 33, No. 12, 1995, pp. 2364-2370.

${ }^{4}$ Valliappan, S. and Pham, T., "Elasto-Plastic Finite Element Analysis with Fuzzy Parameters," International Journal for Numerical Methods in Engineering, Vol. 38, 1995, pp. 531-548.

${ }^{5}$ Moens, D. and Vandepitte, D., "A Survey of Non-Probabilistic Uncertainty Treatment in Finite Element Analysis," Computer Methods in Applied Mechanics and Engineering, Vol. 194, No. 14-16, 2005, pp. 1527-1555.

${ }^{6}$ Moens, D., A Non-Probabilistic Finite Element Approach for Structural Dynamic Analysis with Uncertain Parameters, PhD thesis, K.U.Leuven, Leuven, 2002.

${ }^{7}$ Moens, D. and Vandepitte, D., "An Interval Finite Element Approach for the Calculation of Envelope Frequency Response Functions," International Journal for Numerical Methods in Engineering, Vol. 61, No. 14, 2004, pp. 2480-2507.

${ }^{8}$ Zadeh, L., "Fuzzy sets," Information and Control, Vol. 8, 1965, pp. 338-353.

${ }^{9}$ Moens, D. and Vandepitte, D., "Non-probabilistic approaches for non-deterministic dynamic FE analysis of imprecisely defined structures," Proc. of ISMA 2004, International Conference on Noise and Vibration Engineering, 2004, pp. 3095-3119.

${ }^{10}$ Hanss, M., "The Transformation Method for the Simulation and Analysis of Systems with Uncertain Parameters," Fuzzy Sets and Systems, Vol. 13, 2002, pp. 277-289. 